\title{
CCAAT/enhancer-binding protein alpha (CEBPA) gene haploinsufficiency does not alter hematopoiesis or induce leukemia in Lck-CALM/AF10 transgenic mice
}

\author{
A.P. Lange (ii) ${ }^{1,2 *}$, L.Y. Almeida (ii) ${ }^{1,2 *}$, C.L. Araújo Silva (i) ${ }^{1,2}$, P.S. Scheucher (ii) ${ }^{1,2}$, F. Chahud (ii) ${ }^{3}$ \\ A. Krause (ii) ${ }^{4}$, S.K. Bohlander (10) ${ }^{5}$ and E.M. Rego (ii) $1,2,6$ \\ ${ }^{1}$ Divisão de Hematologia, Departamento de Clínica Médica, Faculdade de Medicina de Ribeirão Preto, \\ Universidade de São Paulo, Ribeirão Preto, SP, Brasi \\ ${ }^{2}$ Centro de Terapia Celular, Faculdade de Medicina de Ribeirão Preto, Universidade de São Paulo, Ribeirão Preto, SP, Brasil \\ ${ }^{3}$ Departamento de Patologia e Medicina Legal, Faculdade de Medicina de Ribeirão Preto, \\ Universidade de São Paulo, Ribeirão Preto, SP, Brasil \\ ${ }^{4}$ Laboratório de Análises Clínicas Veterinárias, Universidade Federal de Santa Maria, Santa Maria, RS, Brasil \\ ${ }^{5}$ Leukaemia \& Blood Cancer Research Unit, Department of Molecular Medicine and Pathology, \\ The University of Auckland, Auckland, New Zealand \\ ${ }^{6}$ Divisão de Hematologia, LIM31, Faculdade de Medicina, Universidade de São Paulo, São Paulo, SP, Brasil
}

\begin{abstract}
Although rare, CALM/AF10 is a chromosomal rearrangement found in immature T-cell acute lymphoblastic leukemia (T-ALL), acute myeloid leukemia, and mixed phenotype acute leukemia of T/myeloid lineages with poor prognosis. Moreover, this translocation is detected in $50 \%$ of T-ALL patients with gamma/delta T cell receptor rearrangement, frequently associated with low expression of transcription factor CCAAT/enhancer-binding protein alpha (CEBPA). However, the relevance of CEBPA low expression for CALM/AF10 leukemogenesis has not yet been evaluated. We generated double mutant mice, which express the Lck-CALM/AF10 fusion gene and are haploinsufficient for the Cebpa gene. To characterize the hematopoiesis, we quantified hematopoietic stem cells, myeloid progenitor cells, megakaryocyte-erythrocyte progenitor cells, common myeloid progenitor cells, and granulocyte-macrophage progenitor cells. No significant difference was detected in any of the progenitor subsets. Finally, we tested if Cebpa haploinsufficiency would lead to the expansion of Mac- $1^{+} / \mathrm{B} 220^{+} / \mathrm{c}-\mathrm{Kit}^{+}$cells proposed as the CALM/AF10 leukemic progenitor. Less than $1 \%$ of bone marrow cells expressed Mac-1, B220, and c-Kit with no significant difference between groups. Our results showed that the reduction of Cebpa gene expression in Lck-CALM/AF10 mice did not affect their hematopoiesis or induce leukemia. Our data corroborated previous studies suggesting that the CALM/AF10 leukemia-initiating cells are early progenitors with lymphoid/myeloid differentiating potential.
\end{abstract}

Key words: Leukemia; CEBPA; CALM/AF10; Transgenic mice

\section{Introduction}

The CALM/AF10 fusion gene derived from the $\mathrm{t}(10 ; 11)$ (p13;q14) chromosomal translocation can be found in Tcell acute lymphoblastic leukemia (T-ALL), acute myeloid leukemia (AML) and mixed phenotype acute leukemia (MPAL) of $T /$ myeloid lineages (1-5). The leukemogenic activity of CALM/AF10 was demonstrated in several murine models. Mice transplanted with murine bone marrow (BM) cells expressing CALM/AF10 fusion transcripts developed an aggressive form of biphenotypic leukemia (6). Accordingly, Caudell et al. (7) generated transgenic mice (TM) expressing CALM/AF10 under the control of the vav promoter (a pan-hematopoietic vector) and approximately $40-50 \%$ of TM developed leukemia at the median age of 12 months. The leukemic blasts isolated from the BM and spleen of vav-CALM/AF10 TM co-expressed Mac-1, $B 220$, and CD24 with variable expression of CD117 in the absence of other B-cell markers, thus resembling human MPAL with involvement of $B$ and myeloid lineages. However, Abdelali et al. (8) screened 665 patients with T-ALL and identified CALM-AF10 in 30/431 (7\%) patients aged 16 years

Correspondence: E.M. Rego: <eduardo.rego@fm.usp.br> <emrego@hcrp.usp.br>

${ }^{*}$ These authors contributed equally to this study. 
and over, and in $15 / 234(6 \%)$ of those aged up to 15 years of age.

Aberrant expression of T-cell lineage markers on AML blasts has been associated with mutations or epigenetic changes of the CEBPA gene or its promoter (9). CEBPA encodes for a transcription factor of crucial relevance for granulocytic differentiation (10). In addition to mutations in the CEBPA gene, which were detected in approximately in $6-15 \%$ of de novo AML and in $15-18 \%$ of $A M L$ with normal karyotypes (11), silencing of CEBPA expression due to promoter hypermethylation was found in a small subset of AML patients with myeloid/T-lymphoid features (12). In the study by Terriou et al. (13), half of the CEBPA methylated T-ALL cases were CALM/AF10 positive (5/10), suggesting that the search of CALM/AF10 in CEBPA methylated AML could help distinguish between the immature T-ALL and myeloperoxidase negative AML. Based on the existing data showing the aberrantly low expression of the CEBPA gene in patients with acute leukemia harboring the CALM/AF10 fusion gene, we decided to evaluate in vivo if haploinsufficiency of CEBPA predisposes CALM/AF10 ${ }^{+}$hematopoietic cells to develop leukemia.

\section{Material and Methods}

\section{Generation of double mutant mice}

Mice haploinsufficient for the Cebpa gene $\left(\mathrm{Cebpa}^{+/-}\right)$ were kindly provided by Prof. Daniel G. Tenen (National University of Singapore) and were crossed to Lck-CALM/ $A F 10 \mathrm{TM}$, in which the expression of the fusion gene is under the control of the lck regulatory region. The LckCALM/AF10 TM were kindly provided by Prof. Stefan $\mathrm{K}$ Bohlander (University of Auckland). The phenotype of parental lines has been previously described (14). The offspring was genotyped by conventional polymerase chain reaction (PCR) (Table 1 ).

\section{Serial analysis of hematological counts}

Animals aged 3-15 months were monitored once every three months for peripheral blood counts. Leishmanstained blood smears were used for differential leukocyte counts. All animal experiments were performed after the approval of the Animal Care and Use Committee of the Medical School of Ribeirão Preto of the University of São Paulo (Protocol number: 144/2013) in strict accordance with institutional guidelines regulated by the National Council of Animal Experimentation (CONCEABrazil). All experimental procedures were performed under isoflurane anesthesia, and all efforts were made to minimize suffering.

\section{Flow cytometry analysis of BM hematopoietic progenitors and mature cells}

Five to seven mice of each group were sacrificed with anesthetic overdose at the age of 15 months and BM single cell suspensions were prepared by crushing femurs, tibiae, and iliac crests with a pestle. The percentage of mature BM cells was identified using murine markers for erythroid precursors (anti-TER-119, clone TER-119), granulocytes (anti-CD11b/Mac-1, clone M1/70), and B (anti-B220, clone RA3-6B2) and T cells (anti-CD3, clone 145-2C11). All antibodies were purchased from $B D$ Pharmingen, USA. To identify the hematopoietic progenitor populations, BM cells were depleted of mature erythroid, myeloid, and lymphoid lineages using the Lineage Cell Detection Cocktail-Biotin, mouse (Miltenyi Biotec, USA). The remaining cells were stained for anti-Ly-6A/Sca-1 (clone D7, Biolegend, USA), anti-CD34 (clone RAM34, eBioscience, USA), CD16/32 (clone 2.4G2, Biolegend), anti-CD117/c-kit (clone 2B8, Biolegend), anti-B220 (clone RA3-6B2, BD Pharmingen), anti-CD3 (clone 145-2C11, BD Pharmingen), anti-Cd11b/Mac-1 (clone M1/70, BD Pharmingen), and anti-TER119 (clone TER-119, BD Pharmingen). The following progenitor populations were quantified: HSC (hematopoietic stem cells, $\mathrm{Lin}^{-} \mathrm{Sca}-1^{+} \mathrm{c}-\mathrm{Kit}^{+}$), MP (myeloid progenitor cells, $\mathrm{Lin}^{-} \mathrm{Sca}-1^{-} \mathrm{c}-\mathrm{Kit}^{+}$), MEP (megakaryocyte-erythrocyte progenitor cells, $\mathrm{Lin}^{-} \mathrm{Sca}-1^{-} \mathrm{C}-\mathrm{Kit}^{+}$ Fcy ${ }^{\text {lo }} \mathrm{CD}^{-} 4^{-}$), CMP (common myeloid progenitor cells, $\mathrm{Lin}^{-} \mathrm{Sca}-1^{-} \mathrm{C}_{-\mathrm{Kit}^{+}} \mathrm{Fcy}^{\mathrm{lo}} \mathrm{CD} 34^{+}$), and GMP (granulocytemacrophage progenitor cells, $\mathrm{Lin}^{-} \mathrm{Sca}-1^{-} \mathrm{C}^{-\mathrm{Kit}^{+}} \mathrm{Fcy}^{\mathrm{hi}}$ CD34 ${ }^{+}$). Samples were analyzed on a JSAN cell sorter (Bay biosciences, Japan) after exclusion of dead cells using DAPI staining. Data were analyzed with FlowJo software (TreeStar, USA).

Table 1. Primers used for mouse genotyping.

\begin{tabular}{|c|c|c|c|c|}
\hline Genotyping reaction & Direction & Primer $\left(5^{\prime}-3^{\prime}\right)$ & Product Size $(\mathrm{pb})$ & Annealing temperature $\left({ }^{\circ} \mathrm{C}\right)$ \\
\hline \multirow[t]{2}{*}{ PCR CALM/AF10 } & $\mathrm{F}$ & 5'-CCAAACTCCCACCTAGCAAGTTAG-3' & 1023 & $57^{\circ} \mathrm{C}, 45 \mathrm{~s}$ \\
\hline & $\mathrm{R}$ & 5'-GGTGTGTGCAGAGACTTCCTG-3' & & 35 cycles \\
\hline \multirow[t]{3}{*}{$\mathrm{PCR}_{\mathrm{Cebpa}}^{(+/-)}$} & $\mathrm{F}$ & 5'-GCCTACCGGTGGATGTGGAATGTG-3' & 300 and 500 & $56^{\circ} \mathrm{C}, 1 \mathrm{~min}$ \\
\hline & $\mathrm{R}$ & 5'-ACTTAGGTGTTGGGGATTTGAGTCTGTG-3' & & 35 cycles \\
\hline & $\mathrm{F}$ & 5'-TCCCCCAGCCGTTAGTGAAGAGTCTC-3' & & \\
\hline \multirow[t]{2}{*}{ PCR beta-actin } & $\mathrm{F}$ & 5'-TCTTGATAGTTCGCCATGGAT-3' & 500 & $64^{\circ} \mathrm{C}, 30 \mathrm{~s}$ \\
\hline & $\mathrm{R}$ & 5'-GGTCATCTTTTCACGGTTGG-3' & & 30 cycles \\
\hline
\end{tabular}

F: forward; R: reverse. 


\section{Histopathological analysis}

Vertebrae and spleens from animals used for flow cytometry analysis were first fixed in PBS with $4 \%$ paraformaldehyde and processed for paraffin-embedded sectioning at $5 \mu \mathrm{m}$ in thickness, and then stained with hematoxylin \& eosin.

\section{Results}

After a median follow-up of 15 months, no significant difference in the median values of leukocyte counts, red blood cell counts, hemoglobin concentrations, and platelet counts were detected among the four genotypes.
Figure $1 \mathrm{~A}-\mathrm{D}$ shows the main hematological parameters of peripheral blood observed in last sample collection during monitoring, but similar results were found at all timepoints. Moreover, there was no change in the percentage of erythroid precursors, granulocytes, and $\mathrm{B}$ and $\mathrm{T}$ lymphocytes in the BM (Figure 1E-H). Of note, no case of leukemia was detected among 23 mice of the $C A^{+} / W T$ group and the 33 animals of the $\mathrm{CA}^{+} / \mathrm{Cebpa}^{+/}$group. The number of animals was adequate for the objectives and followed the good practice guide for the use of animals in research. Our results were in agreement with Krause (14) who did not detect leukemia in Lck-CALM/ AF10 TM.
A

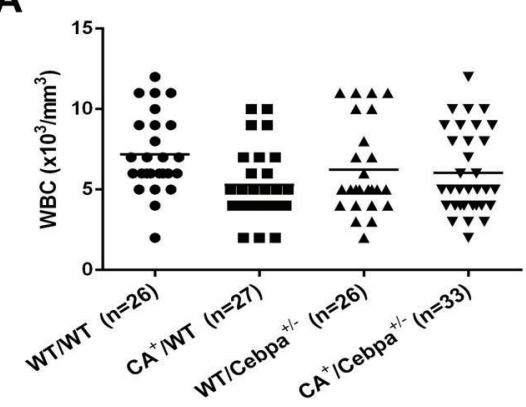

D

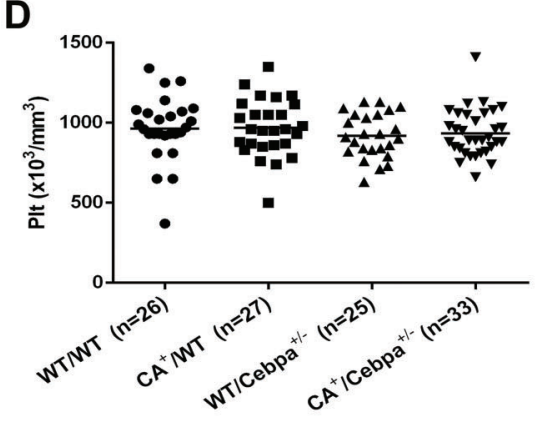

G

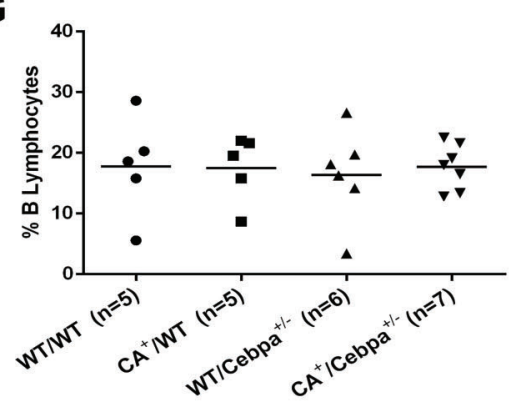

B

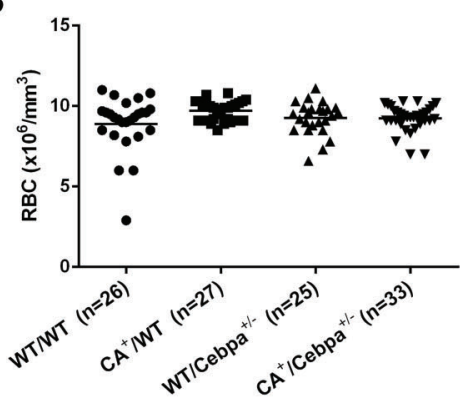

E

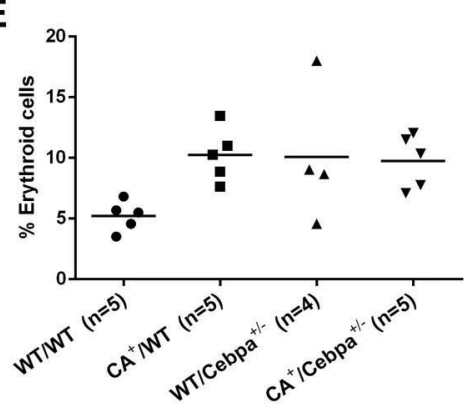

H

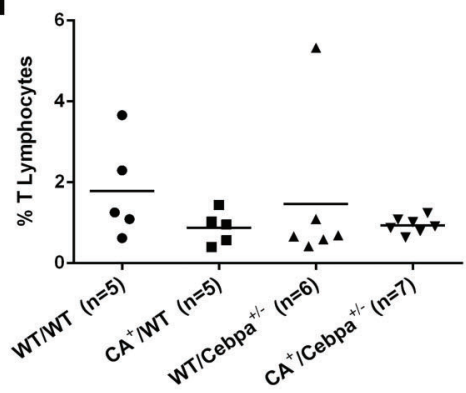

C

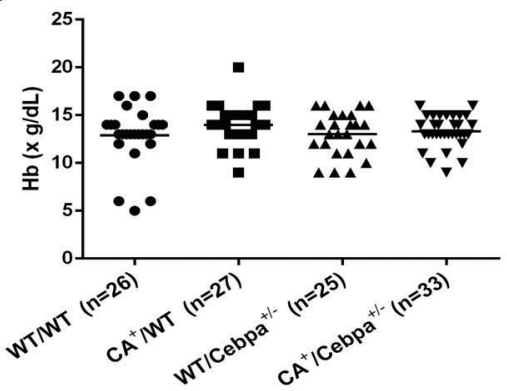

$\mathbf{F}$

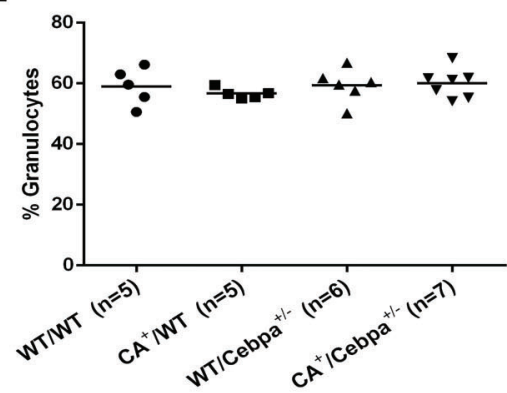

Figure 1. Hematological parameters and immunophenotypic characterization of bone marrow cells of adult mice (9 to 15 months). A, WBC: leukocyte counts. B, RBC: red blood cell counts. C, Hb: hemoglobin concentrations. D, PIt: platelet counts. E, Percentage of erythroid cells. F, Percentage of granulocytes. G, Percentage of B lymphocytes. H, Percentage of T lymphocytes. Each dot represents an individual mouse and lines indicate the median. WTMT: wild-type (control); $C A^{+} / W T: C A L M / A F 10 ; W_{T} / C$ bpa $^{+/-}: \mathrm{Cebpa}^{+/-} ; \mathrm{CA}^{+} /$ $\mathrm{Cebpa}^{+/-}$: double-mutant mice. No significant difference was found between groups. Statistical analysis was performed with the Kruskal-Wallis test followed by Dunn's multiple comparison tests. 
CALM/AF10 rearrangements have been detected in patients with acute leukemia of different phenotypes, but are more frequently associated with T-ALL $(2,5)$. Terriou et al. (15) evaluated CEBPA promoter methylation in $5 \mathrm{AML}$ and 99 T-ALL cases, with expression of cytoplasmic CD3 and CD7 transcripts, T-cell receptor rearrangement, and NOTCH1 mutations. Half of the cases with hypermethylated CEBPA regulatory regions were T-ALL CALM/AF10 positive. These findings suggest that the low expression of CEBPA caused by epigenetic mechanisms may play a role in CALM/AF10 leukemogenesis.
In the present study, CALM/AF10 expression was driven by the $L c k$ proximal promoter region. During normal T-cell ontogenesis, Lck transcription is regulated by two distinct promoter elements. The proximal promoter is active almost exclusively in thymocytes and becomes inactive later during T-cell maturation and has been used experimentally to drive the expression of the transgene to T-cell lineage (16-17). However, Bell and Bhandoola (18) demonstrated that the earliest thymic progenitors have not only lymphoid but also myeloid potential during hematopoiesis, providing alternative phenotypes for a
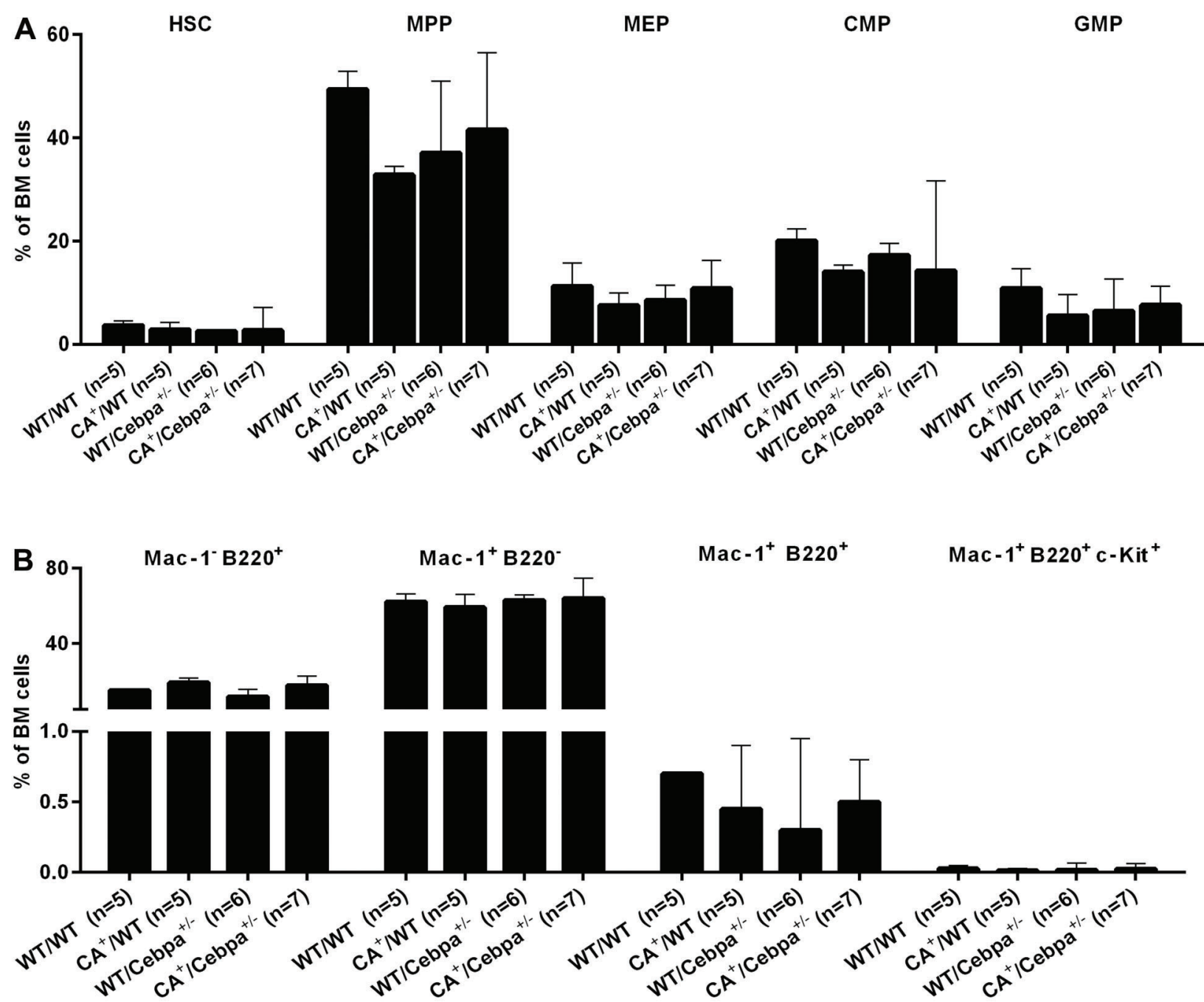

Figure 2. Immunophenotypic characterization of bone marrow (BM) hematopoietic progenitor cells between mice groups. WT/WT: wildtype (control); $C A^{+} / W T: C A L M / A F 10 ; W T / \mathrm{Cebpa}^{+-}$: $\mathrm{Cebpa}^{+/-} ; \mathrm{CA}^{+} / \mathrm{Cebpa}^{+/-}$: double-mutant mice. A, Frequencies of HSC

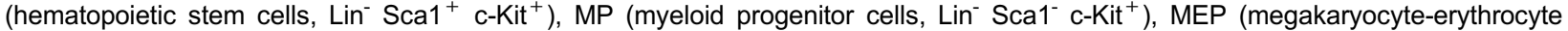
progenitor cells, $\mathrm{Lin}^{-} \mathrm{Sca}^{-} \mathrm{c}-\mathrm{Kit}^{+} \mathrm{Fcy}^{\mathrm{lo}} \mathrm{CD} 34^{-}$), CMP (common myeloid progenitor cells, $\mathrm{Lin}^{-} \mathrm{Sca}^{-} \mathrm{c}-\mathrm{Kit}^{+} \mathrm{Fcy}^{\mathrm{lo}} \mathrm{CD} 34^{+}$), and GMP (granulocyte-macrophage progenitor cells, Lin $^{-} \mathrm{Sca}^{-} \mathrm{c}-\mathrm{Kit}^{+} \mathrm{Fcy}^{\mathrm{hi}} \mathrm{CD}_{4}{ }^{+}$) of WTMT, CA ${ }^{+} / W T$, WT/Cebpa ${ }^{+1-}$, and CA ${ }^{+} / \mathrm{Cebpa}^{+/-}$ mice. Data are reported as means and standard deviations. B, Bone marrow hematopoietic progenitors with features of leukemic stem cells Mac- $1^{-} \mathrm{B} 220^{+}$cells, Mac- $1^{+} \mathrm{B} 220^{-}$cells, Mac- $1^{+} \mathrm{B} 220^{+}$cells, and Mac- $1^{+} \mathrm{B} 220^{+} \mathrm{c}^{-\mathrm{Kit}}{ }^{+}$cells. No significant difference was observed. Statistical analysis was performed with Kruskal-Wallis test followed by Dunn's multiple comparison tests. 
lineage specification of a potential leukemic transformation driven by an Ick-CALM/AF10 translocation. In our model, CALM/AF10 expression occurred in hematopoietic progenitors with restricted capacity of differentiation and our results suggest that CALM/AF10 may not be leukemogenic in this cell compartment. Reinforcing this hypothesis, Caudell et al. (7) generated a murine model in which the expression of CALM/AF10 was targeted to multipotent progenitors using the vav promoter, and reported that approximately $50 \%$ of vav-CALM/AF10 TM developed leukemia. The leukemic blasts showed myeloid differentiation associated with lymphoid features, thus resembling human MPAL. However, the long latency of the disease in this model further argues that additional events are necessary for the development of leukemia.

\section{Discussion}

In order to fully characterize the hematopoiesis in WTIWT, $\mathrm{CA}^{+} / \mathrm{WT}, \mathrm{WT} / \mathrm{Cebpa}^{+/-}, \mathrm{CA}^{+} / \mathrm{Cebpa}^{+/-}$mice,

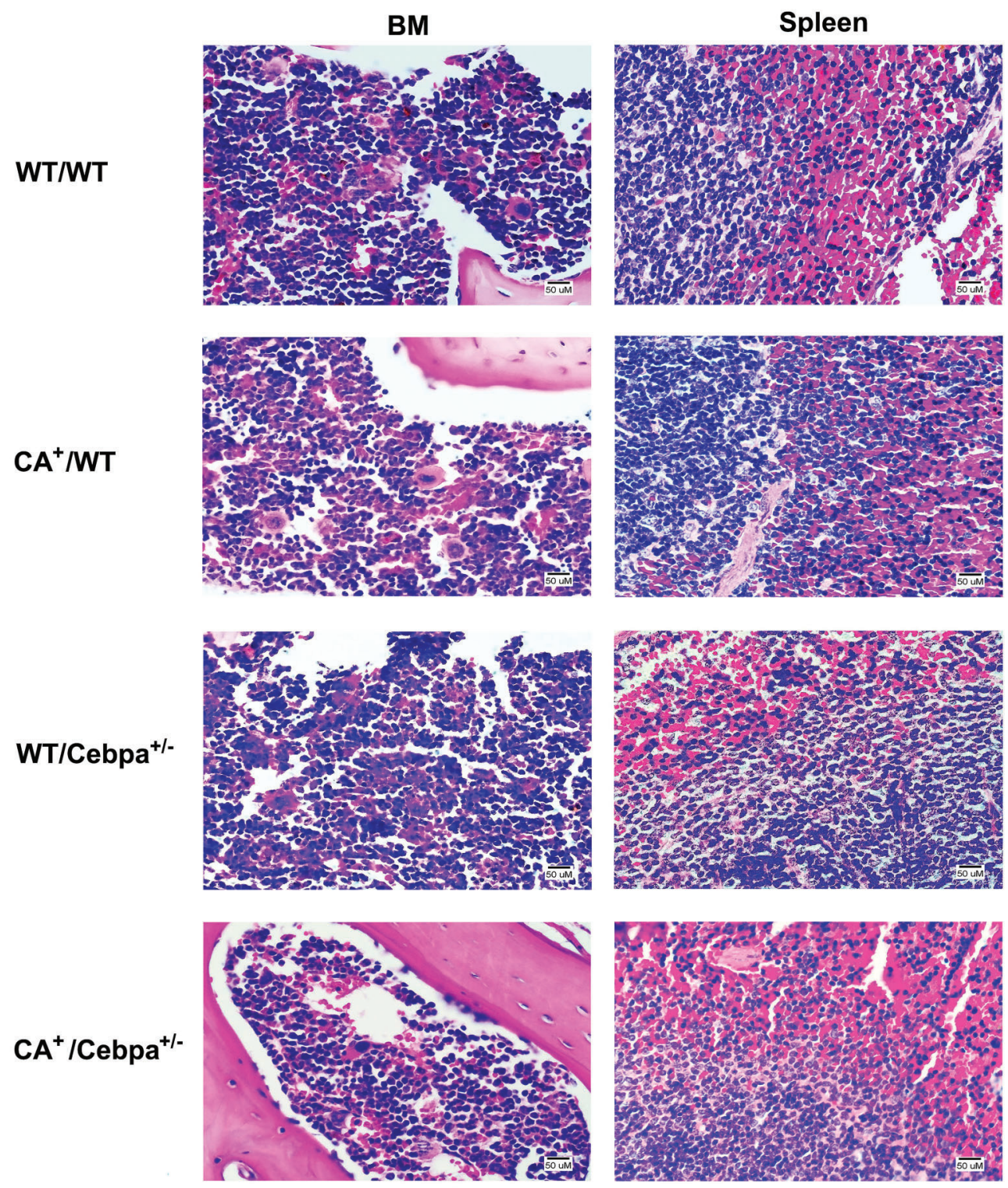

Figure 3. Histopathological analysis of the bone marrow (BM) and spleen from WTWT: wild-type (control); $C A^{+} / W T$ : $C A L M / A F 10$; $W T / \mathrm{Cebpa}^{+l-}$ : $\mathrm{Cebpa}^{+1-} ; \mathrm{CA}^{+} / \mathrm{Cebpa}^{+l-}$ : double-mutant mice. The normal architecture of the bone marrow and spleen was preserved in $C A^{+} / W T, W T / \mathrm{Cebpa}^{+/-}$, and $\mathrm{CA}^{+} / \mathrm{Cebpa}^{+/-}$mice and showed no changes in the cellularity pattern compared to control mice. Hematoxylin and eosin stain. Bar: $50 \mu \mathrm{m}$. 
we examined whether there were genotype-specific differences in BM HSC and myeloid progenitors (MP, MEP, CMP, and GMP). Frequencies of HSC, MP, MEP, CMP, and GMP did not differ between groups (Figure 2A). In addition, BM and spleen did not show differences between the groups on histopathological analysis (Figure 3).

Decreased expression of CEBPA plays a role in acute promyelocytic leukemia (APL) leukemogenesis. Guibal et al. reported that $\mathrm{Cebpa}^{+/-}$mice did not develop leukemia nor present abnormal cell counts, but double mutants human cathepsin G (hCG)-PML/RARA Cebpa ${ }^{+/-}$developed APL at a higher frequency, decreased latency, and had a significantly shorter survival rate than hCG-PML/ RARA TM. Furthermore, the APL-initiating cells showed decreased mRNA and protein expression of CEBPA compared with normal promyelocytes (19).

Deshpande et al. (6) showed that, in a transplantation model of murine CALM/AF $10^{+}$leukemia, the myeloid leukemia cells were frequently $B 220^{+}$, with clonal $B$ cell receptor $(B C R)$ rearrangement and lacked $\mathrm{c}-$ Kit expression, demonstrating that these AML cells had lymphoid characteristics. In contrast, in the study of Caudell et al. (7), only $50 \%$ of the AML from vav-CALM/AF10 TM were $\mathrm{B}_{2} 20^{+}$and exhibited clonal BCR rearrangement, with variable expression of c-Kit. Nevertheless, both studies showed that $\mathrm{Mac}-1^{+} / \mathrm{B} 220^{+}$cells lacked specific B-cell markers. Dutta et al. (20) studied the role of CALM/AF10 specifically in $B$ cell development and function in Mb1-Cre or CD19-Cre mice and demonstrated that expression of CALM/AF10 in the B cell compartment did not lead to leukemia. Together, these authors show evidence that the

\section{References}

1. Kumon K, Kobayashi H, Maseki N, Sakashita A, Sakurai M, Tanizawa A, et al. Mixed-lineage leukemia with $\mathrm{t}(10 ; 11)(\mathrm{p} 13$; q21): an analysis of AF10-CALM and CALM/AF10 fusion mRNAs and clinical features. Genes Chromosomes Cancer 1999; 25: 33-39, doi: 10.1002/(SICI)1098-2264(199905) 25:1<33::AID-GCC5 > 3.0.CO;2-3.

2. Groupe Français de Cytogénétique Hématologique. $t(10 ; 11)$ (p13-14;q14-21): a new recurrent translocation in T-cell acute lymphoblastic leukemias. Genes Chromosomes Cancer 1991; 3: 411-415, doi: 10.1002/gcc.2870030602.

3. Groupe Français de Cytogénétique Hématologique. Collaborative study of karyotypes in childhood acute lymphoblastic leukemias. Leukemia 1993; 7: 10-19.

4. Dreyling $\mathrm{MH}$, Schrader K, Fonatsch $\mathrm{C}$, Schlegelberger B, Haase D, Schoch C, et al. MLL and CALM are fused to AF10 in morphologically distinct subsets of acute leukemia with translocation $\mathrm{t}(10 ; 11)$ : both rearrangements are associated with a poor prognosis. Blood 1998; 91: 4662-4667.

5. Bohlander SK, Muschinsky V, Schrader K, Siebert R, Schlegelberger $B$, Harder $L$, et al. Molecular analysis of the CALM/AF10 fusion: identical rearrangements in acute myeloid leukemia, acute lymphoblastic leukemia and malignant lymphoma patients. Leukemia 2000; 14: 93-99, doi: 10.1038/ sj.leu.2401614. cell of origin of CALM/AF10 AML with lymphoid characteristics is an early progenitor not derived from B-cell lineage. In order to test if there is an expansion of the rare population of hematopoietic progenitor cells (Mac- $1^{+} /$ $\mathrm{B}^{2} 20^{+}$) followed or not by the expression of c-Kit in the $\mathrm{BM}$ of double mutant mice $\mathrm{CA}^{+} / \mathrm{Cebpa}^{+/-}$, we quantified the frequency of these cells in our model. Less than $1 \%$ of BM cells expressed Mac-1, B220, and c-Kit with no significant difference between groups (Figure 2B).

In summary, our results showed that the reduction of Cebpa expression did not contribute to leukemogenesis in Lck-CALM/AF10 mice and did not affect hematopoiesis. In future experiments, it will be important to determine if decreased Cebpa expression can modify leukemia frequency or promote the expansion of specific cell subsets in vav-CALM/AF10/Cebpa ${ }^{+/-}$double mutant mice. Nevertheless, our data support the hypothesis that the normal counterpart of the CALM/AF10 ${ }^{+}$leukemia-initiating cells is a hematopoietic stem cell or an early progenitor with myeloid and lymphoid potential.

\section{Acknowledgments}

This work was supported by the Fundação de Apoio à Pesquisa do Estado de São Paulo (FAPESP; Grant No. 2013/08135-2). A.P.L. and L.Y.A. received FAPESP fellowships (Grant Nos. 2011/17111-4 and 2016/02713-2, respectively). This work was supported by SFB 684 project A6. S.K.B. is supported by Leukaemia \& Blood Cancer New Zealand and the family of Marijana Kumerich.

6. Deshpande AJ, Cusan M, Rawat VP, Reuter H, Krause A, Pott $C$, et al. Acute myeloid leukemia is propagated by a leukemic stem cell with lymphoid characteristics in a mouse model of CALM/AF10-positive leukemia. Cancer Cell 2006; 10: 363-374, doi: 10.1016/j.ccr.2006.08.023.

7. Caudell D, Zhang Z, Chung YJ, Aplan PD. Expression of a CALM/AF10 fusion gene leads to Hoxa cluster overexpression and acute leukemia in transgenic mice. Cancer Res 2007; 67: 8022-8031, doi: 10.1158/0008-5472.CAN06-3749.

8. Ben Abdelali R, Asnafi V, Petit A, Micol JB, Callens C, Villarese $P$, et al. The prognosis of CALM-AF10-positive adult T-cell acute lymphoblastic leukemias depends on the stage of maturation arrest. Haematologica 2013; 98: 17111717, doi: 10.3324/haematol.2013.086082.

9. Wouters BJ, Koss C, Delwel R. Gene expression profiling for improved dissection of acute leukemia: a recently identified immature myeloid/T-lymphoid subgroup as an example. Blood Cells Mol Dis 2008; 40: 395-400, doi: 10.1016/j.bcmd. 2007.10.006.

10. Ohlsson E, Schuster MB, Hasemann M, Porse BT. The multifaceted functions of $\mathrm{C} / \mathrm{EBP} \alpha$ in normal and malignant haematopoiesis. Leukemia 2016; 30: 767-775, doi: 10.1038/ leu.2015.324. 
11. Bienz M, Ludwig M, Leibundgut EO, Mueller BU, Ratschiller D, Solenthaler M, et al. Risk assessment in patients with acute myeloid leukemia and a normal karyotype. Clin Cancer Res 2005; 11: 1416-1424, doi: 10.1158/1078-0432.CCR-04-1552.

12. Wouters BJ, Jordà MA, Keeshan K, Louwers I, ErpelinckVerschueren CA, Tielemans D, et al. Distinct gene expression profiles of acute myeloid/T-lymphoid leukemia with silenced CEBPA and mutations in NOTCH1. Blood 2007; 110: 3706-3714, doi: 10.1182/blood-2007-02-073486.

13. Terriou L, Ben Abdelali R, Roumier $C$, de Vos J, Cornillet $P$, Nibourel $\mathrm{O}$, et al. CEBPA methylation and Notch1 mutations are common in immature T-ALLs but not MO AML and cosegregate with TCRG rearrangement and the TCRgd lineage. Blood 2007; 110: 2788.

14. Krause A. Analysis of the leukemogenic potential of the CALM/AF10 Fusion gene in patients, transgenic mice and cell culture models. [PhD Thesis]. Munich: Ludwig-MaximiliansUniversity of Munich; 2006.

15. Terriou L, Ben Abdelali R, Roumier C, Lhermitte L, de Vos J, Cornillet $\mathrm{P}$, et al. C/EBPA methylation is common in T-ALL but not in MO AML. Blood 2009; 113: 1864-1866, doi: 10.1182/ blood-2008-09-176909.
16. Allen JM, Forbush KA, Perlmutter RM. Functional dissection of the Ick proximal promoter. Mol Cell Biol 1992; 12: 27582768, doi: 10.1128/MCB.12.6.2758.

17. Shimizu C, Kawamoto H, Yamashita M, Kimura M, Kondou $\mathrm{E}$, Kaneko $\mathrm{Y}$, et al. Progression of $\mathrm{T}$ cell lineage restriction in the earliest subpopulation of murine adult thymus visualized by the expression of Ick proximal promoter activity. Int Immunol 2001; 13: 105-117, doi: 10.1093/ intimm/13.1.105.

18. Bell JJ, Bhandoola A. The earliest thymic progenitors for T cells possess myeloid lineage potential. Nature 2008; 452: 764-767, doi: 10.1038/nature06840.

19. Guibal FC, Alberich-Jorda M, Hirai H, Ebralidze A, Levantini $E$, Di Ruscio A, et al. Identification of a myeloid committed progenitor as the cancer-initiating cell in acute promyelocytic leukemia. Blood 2009; 114: 5415-5425, doi: 10.1182/blood2008-10-182071.

20. Dutta S, Krause A, Vosberg S, Herold T, Ksienzyk B, Quintanilla-Martinez $\mathrm{L}$, et al. The target cell of transformation is distinct from the leukemia stem cell in murine CALM/AF10 leukemia models. Leukemia 2016; 30: 1166-1176, doi: 10.1038/leu.2015.349. 